\title{
Unusual Presentation and Imaging Findings of a Rare Supratentorial Neurenteric Cyst
}

\author{
Eduardo Portela de Oliveira $₫$, John Woulfe, Carlos Torres, Nader Zakhari $®$
}

Keywords: Brain tumours, Neurenteric cyst, Neuroenteric, Intracranial cysts, Brain MRI

doi:10.1017/cjn.2019.295

Can J Neurol Sci. 2020; 47: 121-123

This is a case of a 30-year-old right-handed male patient who presented to the hospital in 2014 after hitting his head on the mat during a wrestling match followed by headache and temporary peripheral vision limitation. The patient's past medical history was unremarkable. On physical examination, Glasgow Coma Scale was 15 with no focal neurological deficits. Unenhanced head computed tomography (CT) and enhanced brain magnetic resonance imaging (MRI) were performed (Figure 1). The patient was managed conservatively, and follow-up CT and MRI in 2015 (Figure 2) demonstrated significant decrease in size of the previously seen right frontoparietal lesion with also changes in its radiological features. The patient remained asymptomatic for about 3 years when in 2018 he presented to the Emergency Department with increasing headaches and peripheral vision loss. MRI demonstrated an increase in the right frontal lesion size (Figure 3). He underwent surgical resection of the lesion.

Neurenteric cysts (NC), also called enterogenous cysts, are rare benign developmental cysts of endodermal origin that may affect the central nervous system. ${ }^{1,2}$ They are most commonly located along the spinal canal as intradural extramedullary cysts. Intracranial occurrences are uncommon and are mostly found in the posterior fossa located near the midline with only fewer than 50 cases reported in the supratentorial compartment. ${ }^{3,4}$ Among the supratentorial cases, there is a predilection for the frontoparietal region and the majority of the reported cases were extra-axial. ${ }^{4,5}$ They are found as large lesions in patients aged between 20 and 50 years, who usually present with headaches, seizures, and motor deficit. $^{2,4}$

The diagnosis of supratentorial NC is challenging due to the broad imaging spectrum correlating with the presence of mucin-producing cells in the lesion. ${ }^{2}$ The differential diagnosis includes arachnoid cyst, epidermoid cyst, dermoid cyst, cystic neoplasms, brain abscess, and parasitic or larval cyst. ${ }^{2,6}$ On imaging, a large hyperdense/isodense cyst on CT, outside of the midline, and the presence of lobulations and septa may aid in formulating a correct diagnosis. This cyst content does not follow the cerebrospinal fluid (CSF) signal intensity on MRI and demonstrates hyperintensity on FLAIR sequence, which is an important feature to distinguish it from an arachnoid cyst, which typically follows the CSF signal intensity on all sequences. ${ }^{7}$ The cystic mass does not restrict diffusion and usually does not present calcifications or fat. The presence of intracystic hemorrhage was indeed a confounding effect and to the best of our knowledge, this is the first case reported in the literature of traumatic hemorrhage involving a supratentorial NC. Traumatic intracystic hemorrhage of arachnoid cysts has been reported occasionally and may complicate the MR appearance. ${ }^{8}$ The absence of any solid-enhancing component and the presence of only mild rim enhancement at the site of attachment to the brain parenchyma can generally exclude entities such as neoplasms or infectious cysts. $^{2}$

\section{CONFLict OF INTEREST}

The authors have no conflicts of interest to declare.

\section{Statement of Authorship}

We confirm that each author has participated sufficiently in this submission, taking public responsibility for its content, and has approved this submission.

EPO: Organization of the images, review of the literature, preparation of manuscript.

JW: Review of the pathology.

CT: Selection of images and clinical review.

NZ: Review of the material and manuscript.

From the Department of Radiology - University of Ottawa, Medical Imaging Division The Ottawa Hospital, Ottawa, ON, Canada (EPO, CT, NZ); Department of Pathology, University of Ottawa, Eastern Ontario Regional Laboratory Association (EORLA); Department of Laboratory Medicine, Ottawa Hospital, Ottawa, ON, Canada (JW)

Received July 18, 2019. Final Revisions Submitted August 23, 2019. Date of Acceptance September 5, 2019.

Correspondence to: Dr. Nader Zakhari, University of Ottawa, Department of Radiology, 501 Smyth Road, Box 232, Ottawa, Ontario, Canada K1H 8L6. Email: nzakhari@toh.ca 

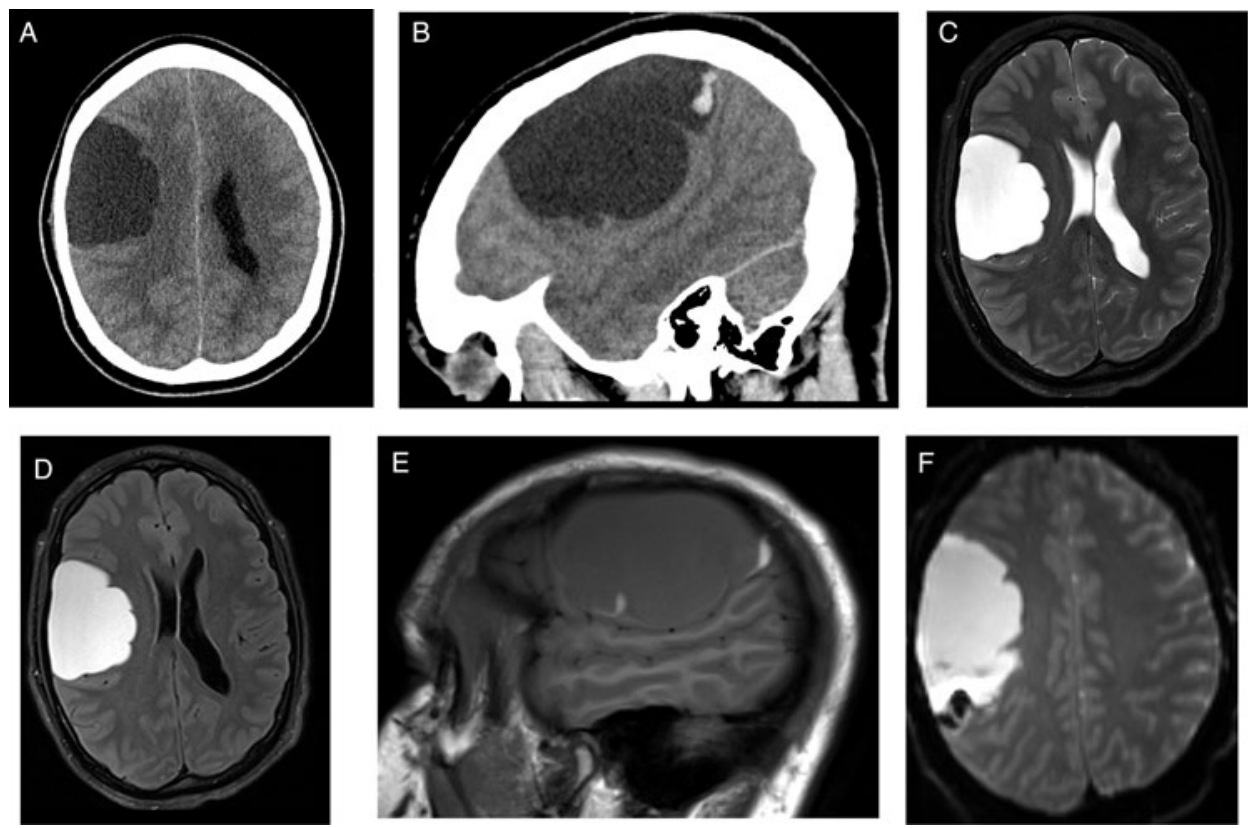

Figure 1: Unenhanced computed tomography $(C T)$ axial $(A)$ and sagittal $(B)$ performed in 2014 revealed a large extra-axial frontoparietal lobulated cystic mass. Magnetic resonance imaging (MRI) axial T2-weighted image $(C)$ showed hyperintense cystic lesion causing mass effect over the brain parenchyma. On fluid-attenuated inversion recovery $(F L A I R)(D)$, the lesion presented higher signal in relation to the cerebrospinal fluid (CSF). Sagittal T1-weighted image $(E)$ showed isointense central content with a focus of hyperintensity in the posterior aspect of the cystic mass. Gradient echo (GRE) images $(F)$ showed blooming artifacts confirming the presence of blood products. No abnormal enhancement or restricted diffusion was seen (not shown).
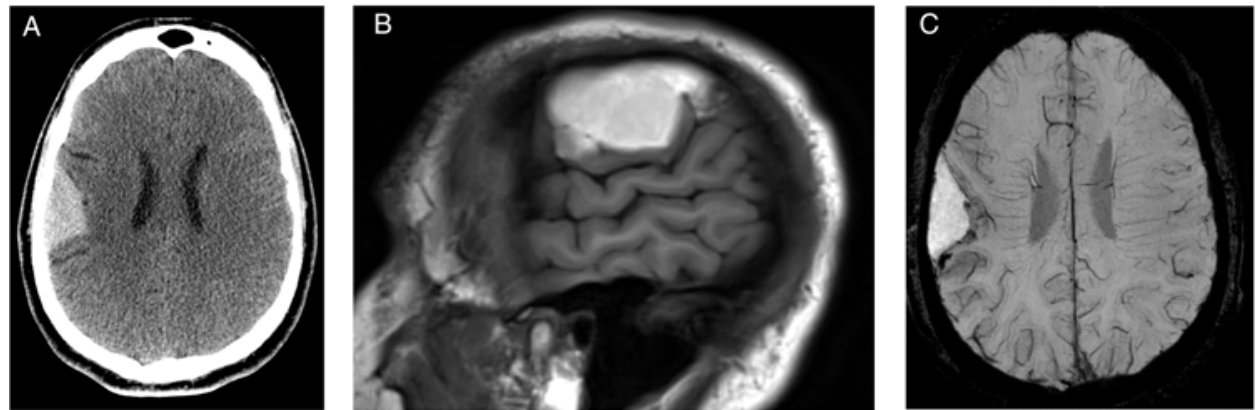

Figure 2: Follow-up CT and MRI after 1 year: Axial CT (A) and MRI sagittal T1-weighted images (B) demonstrated a decrease in size and changes in the internal content of the cystic lesion, which became completely hyperdense on CT and hyperintense on MRI T1-weighted images. Axial susceptibility-weighted imaging (SWI) demonstrated a rim of blooming artifact in the periphery of the lesion, likely secondary to previous hemorrhage $(C)$.
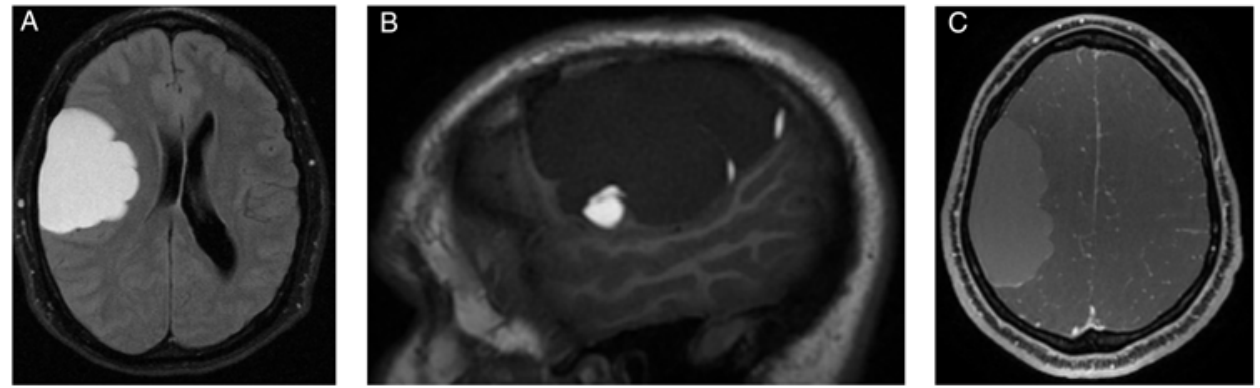

Figure 3: Follow-up MRI after 3 years when the patient became symptomatic: Axial FLAIR (A) demonstrates an increase in size of the cystic lesion. On sagittal T1-weighted images $(B)$, the content of lesion became hypointense with some remaining areas of increased signal in the periphery of the lesion. Axial $T 1$ postcontrast showed areas of minimal linear enhancement $(C)$. 


\section{REFERENCES}

1. Mittal S, Petrecca K, Sabbagh AJ, et al. Supratentorial neurenteric cysts-A fascinating entity of uncertain embryopathogenesis. Clin Neurol Neurosurg. 2010;112(2):89-97. doi:10.1016/j.clineuro. 2009.11.001.

2. Preece MT, Osborn AG, Chin SS, Smirniotopoulos JG. Intracranial neurenteric cysts: imaging and pathology spectrum. Am J Neuroradiol. 2006;27(6):1211-6. www.ajnr.org/content/ $27 / 6 / 1211$.

3. Santos de Oliveira R, Cinalli G, Roujeau T, Sainte-Rose C, PierreKahn A, Zerah M. Neurenteric cysts in children: 16 consecutive cases and review of the literature. $J$ Neurosurg Pediatr. 2005;103(6):512-23. doi:10.3171/ped.2005.103.6.0512.

4. Góes P, Vaz-Guimaraes F, Suriano IC, Araújo S, Zymberg ST. Supratentorial neurenteric cyst: analysis of 45 cases in the literature. Interdiscip Neurosurg Adv Tech Case Manag. 2018; 11:57-64. doi:10.1016/j.inat.2017.08.008.

5. Rangarajan V, Mahore A, Patil MK, Shendarkar AD. Supratentorial endodermal cysts - Report of two cases. Asian J Neurosurg. 2016;11(3):310. doi:10.4103/1793-5482.179642.

6. Bao X-J, Li X-Y, Wang Q-P, et al. Intraparenchymal endodermal cyst with spontaneous intracystic hemorrhage in the temporal lobe of an adult. Medicine (Baltimore). 2016;95(46):e4968. doi:10.1097/ MD.0000000000004968.

7. Osborn AG, Preece MT. Intracranial cysts: radiologic-pathologic correlation and imaging approach. Radiology. 2007;239(3): 650-64. doi:10.1148/radiol.2393050823.

8. De K, Berry K, Denniston S. Haemorrhage into an arachnoid cyst: a serious complication of minor head trauma. Emerg Med J. 2002;19(4):365-6. doi:10.1136/EMJ.19.4.365. 